\title{
Testimoniales en la publicidad radiofónica de productos relacionados con la salud en España y su regulación (2009-2016)
}

\section{Testimonials in health-related radio advertising in Spain and their regulation (2009-2016)}

\author{
Salvador Perelló-Oliver; Clara Muela-Molina; María-Victoria Campos-Zabala
}

Cómo citar este artículo:

Perelló-Oliver, Salvador; Muela-Molina, Clara; Campos-Zabala, María-Victoria (2019). “Testimoniales en la publicidad radiofónica de productos relacionados con la salud en España y su regulación (2009-2016)". El profesional de la información, v. 28, n. 2, e280202.

https://doi.org/10.3145/epi.2019.mar.02

Artículo recibido el 05-10-2018 Aceptación definitiva: 17-11-2018

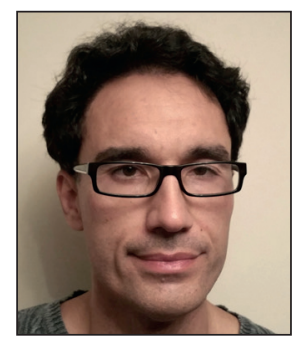

Salvador Perelló-Oliver https://orcid.org/0000-0003-2655-440X

Universidad Rey Juan Carlos

Facultad de Ciencias de la Comunicación

Departamento de Ciencias de la

Comunicación y Sociología

Camino del Molino, s/n. 28943 Fuenlabrada

(Madrid), España

salvador.perello@urjc.es

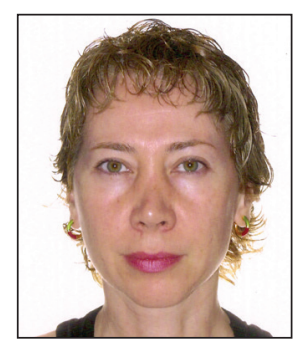

Clara Muela Molina $\square$

https://orcid.org/0000-0002-1344-8731

Universidad Rey Juan Carlos

Facultad de Ciencias de la Comunicación

Departamento de Ciencias de la Comunicación y Sociología

Camino del Molino, s/n. 28943 Fuenlabrada

(Madrid), España

clara.muela@urjc.es

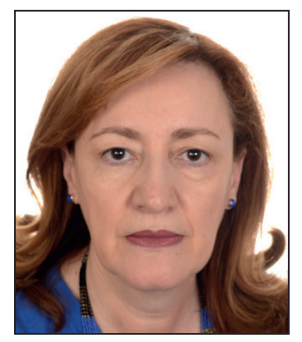

María-Victoria Campos-Zabala

https://orcid.org/0000-0002-6695-6692

Universidad Rey Juan Carlos

Facultad de Ciencias de la Comunicación

Departamento de Ciencias de la Comunicación y

Sociología

Camino del molino, s/n. 28943 Fuenlabrada,

(Madrid), España

mariavictoria.campos@urjc.es

\section{Resumen}

El objetivo de este trabajo es analizar qué perfiles de testimoniales publicitan productos con supuestos beneficios para la salud, las características del testimonio y evolución en los últimos años en la radio española. El estudio, de naturaleza cuantitativa y polietápica, se basa en el análisis de contenido de cuñas publicitarias de dos muestras temporales: 1.664 cuñas en 2009 y 3.909 en 2016, que tras el correspondiente filtrado quedó integrado por 147 y 122 testimoniales, respectivamente. Una de las implicaciones derivadas del trabajo es que la administración pública debe mejorar la monitorización y el control sobre la publicidad ilícita de productos relacionados con la salud. También los propios medios de comunicación mediante protocolos de revisión previa y autorregulación.

\section{Palabras clave}

Publicidad; Radio; Autorregulación; Personificación; Regulación; Salud; Testimoniales.

\section{Financiación}

Esta investigación ha sido desarrollada en el marco del proyecto Responsabilidad social, autorregulación publicitaria y salud en la radio, código (CSO2017-82267-R) financiado por el Ministerio de Economía, Industria y Competitividad, Secretaría de Estado de Investigación, Desarrollo e Innovación. 


\begin{abstract}
The objective of this work is to analyze the nature of testimonials that advertise products with alleged health benefits, the testimonial characteristics and the evolution in Spanish radio. The study of quantitative and multistage nature is based on the content analysis of radio spots from two different time samples: 1,664 spots in 2009 and 3,909 in 2016 which, after the required data filtering process, were reduced to 147 and 122 testimonials, respectively. One of the implications derived from this work is that the public administration should improve the monitoring and control of illicit advertising that involves health-related products. The media itself should implement advertising protocols that include prior review and self-regulation.
\end{abstract}

\title{
Keywords
}

Advertising; Radio; Self-regulation; Personification; Regulation; Health; Testimonials.

\section{Introducción}

La personificación en publicidad -o uso de terceras personas que representan al anunciante- es un objeto de estudio que ha recibido mucha atención por parte de los investigadores desde diversos ámbitos, en especial, para demostrar que su inclusión en el mensaje publicitario incrementa su eficacia (Biswas; Biswas; Das, 2006; Eisend; Langner, 2010; Gaied; Ben-Rached, 2010). Estas personas, llamadas portavoces, pueden ser desde personajes famosos, hasta consumidores corrientes que relatan su experiencia con el producto o servicio anunciado (Friedman; Termini; Washington, 1976), pasando por expertos profesionales y empleados de la propia empresa anunciante.

En España no hay un marco normativo específico que regule el uso de testimoniales en publicidad. Sin embargo, la mayoría de las leyes que regulan la publicidad y el envasado de productos relacionados con la salud establecen limitaciones respecto a su presencia. En concreto, la Ley 17/2011, de 5 de julio, de seguridad alimentaria y nutrición en su Artículo 44, titulado "Publicidad de alimentos", prohíbe expresamente en la publicidad o promoción directa o indirecta de alimentos

"la aportación de testimonios de profesionales sanitarios o científicos, reales o ficticios, o de pacientes reales o supuestos, como medio de inducción al consumo, así como la sugerencia de un aval sanitario o científico" (Espa$\tilde{n} a, 2011)$.

La citada ley también remite a otra normativa específica anterior que regula todos los productos que ofrecen beneficios para la salud exceptuando los medicamentos dispensados sin receta médica. Ello incluye bebidas, alimentos, productos de belleza e higiene, adelgazantes o complementos alimenticios. En el Real Decreto 1907/1996, de 2 de agosto, sobre publicidad y promoción comercial de productos, actividades o servicios con pretendida finalidad sanitaria en cuyo Artículo 4 de "Prohibiciones y limitaciones de la publicidad con pretendida finalidad sanitaria", el punto 7 recoge la prohibición de

"aportar testimonios de profesionales sanitarios, de personas famosas o conocidas por el público o de pacientes reales o supuestos como medio de inducción al consumo" (España, 1996).

Por otro lado, estudios recientes sobre las reclamaciones en materia de publicidad muestran que los productos que proponen beneficios relacionados con la salud son los que han recibido más reclamaciones en los últimos años, seguidos de los de alimentación (Perelló-Oliver; Muela-Molina; Hormigos-Ruiz, 2016) en Autocontrol, el organismo encargado de la autorregulación del sector publicitario. En concreto, las reclamaciones sobre la publicidad de productos alimenticios han pasado de 18 en 2013 y 2014 a 33 en 2015, mientras que las relativas a la categoría de salud se han incrementado en 21 , 45 y 48 en 2013, 2014 y 2015 respectivamente (Perelló-Oliver; Muela-Molina, 2016). El caso de reputados periodistas anunciando productos cuya publicidad fue reclamada y declarada engañosa en muchos casos es un hecho que merece un análisis en mayor profundidad. Cabe añadir que el código ético de los periodistas no permite que éstos colaboren en campañas publicitarias para evitar conflictos de intereses o empañar la transparencia del profesional y del medio (Muela-Molina; Martín-Santana; Reinares-Lara, 2018).

El principal interés de este trabajo es analizar el comportamiento del anunciante respecto a una práctica no permitida en unos productos con alta implicación que, al ser importantes psicológicamente para el consumidor (Buchholz; Smith, 1991), en algunos casos pueden suponer un riesgo para su salud (Peiró-Abásolo, 2006).

\subsection{Revisión de la bibliografía y preguntas de investigación}

La bibliografía que analiza el uso de testimoniales en publicidad ha empleado por lo general el modelo de la credibilidad de la fuente (Ohanian, 1990), demostrando que el aval de un experto o un personaje no famoso es más eficaz que el realizado por una celebridad (Biswas; Biswas; Das, 2006; Eisend; Langner, 2010; Gaied; Ben-Rached, 2010).

Otros trabajos se han basado en el modelo de transferencia de significado (the meaning transfer model) (Amos; Holmes; Strutton, 2008; Doss, 2011; Silvera; Austad, 2004), que se fundamenta en que los famosos aportan un valor añadido a través de dicho proceso de transferencia en contraste con un modelo o actor anónimo, ya que los personajes famosos aportan un significado extra de sutileza, profundidad y poder (McCracken, 1989).

Según el Elaboration likelihood model (ELM), la presencia de determinados testimoniales en publicidad se ha convertido en un objeto muy regulado por parte de la administración pública dado su potencial persuasivo y su incremento en la 
eficacia publicitaria como una variable periférica (Petty; Cacioppo; Schumann, 1983). Kertz y Ohanian (1992) sostienen que el uso efectivo de testimonios en publicidad requiere una comprensión de los principios legales impuestos a su presencia en mensajes persuasivos. En este sentido, la Federal Trade Commission (FTC) ha desarrollado una guía muy completa sobre el correcto uso de testimoniales en publicidad explicando las características, modalidades y diferentes situaciones a través de ejemplos y casos (FTC, 2009). En esta guía se define testimonial como

"cualquier mensaje publicitario (incluyendo declaraciones verbales, demostraciones o representaciones del nombre, firma, semblanza u otras características personales identificativas de un individuo o el nombre o sello de una organización) que los consumidores puedan creer que refleja las opiniones, creencias, hallazgos, o experiencias de un tercero que no sea el anunciante patrocinador" (FTC, 2009, p. 53124).

Según Hastak y Mazis (2011), un testimonio avalado por un experto o un consumidor está sesgado y, por lo tanto puede inducir a engaño cuando la opinión se realiza sobre un asunto o tema alejado de su ámbito de especialización.

En la publicidad de productos relacionados con la salud, trabajos previos han mostrado que el testimonial de un experto (farmacéutico o doctor) o consumidor es más eficaz que el de un famoso (LaTour; Smith, 1986; Limbu; Huhmann; Peterson, 2012; Wu et al., 2012). La intención del anunciante al utilizar personas que hablan en su nombre es transmitir la confianza percibida y crear una imagen para la marca a través de la persona más que a través del producto en sí (Kertz; Ohanian, 1992).

Priester y Petty (2003) sostienen que si un receptor tiene la creencia y seguridad de que una fuente experta está dispuesta a proporcionar información precisa debido a la confianza otorgada al emisor, aquél puede renunciar a la tarea de escudriñar el mensaje y, en su lugar, aceptar la conclusión como válida de manera irreflexiva.

El análisis del tipo de mensaje mostrará la forma en que el portavoz se dirige a la audiencia para describir un producto, es decir, el rol que adopta según el pronombre que utiliza (Khatri, 2006; Seno; Lukas, 2007). Para Keel y Nataraajan (2012), la percepción de la implicación del portavoz en el mensaje ejerce más influencia sobre las actitudes de los consumidores y la intención de compra que la propia implicación en sí. El uso del "yo" revela detalles personales sobre el testimonial. Lo que el "yo" dice se supone que es "verdadero" porque es una revelación sincera de un presentador que no tiene ninguna razón para mentir (Tulloch, 2014). Mientras, la tercera persona es

"una fuente autorizada que procede tanto de la omnisciencia como de la imparcialidad (sabe todo, pero no está directamente involucrado)" (Stern, 1991, p. 13).

Aunque el pronombre utilizado es uno de los principales indicadores de la implicación del testimonial con la marca anunciada, también lo es instar al oyente para que actúe en una forma determinada respecto al producto cuando al finalizar el mensaje utiliza el modo imperativo para pedir algo (McCracken, 1989; Seno; Lukas, 2007). Es una interacción parasocial conativa que se produce en el ámbito del representante del emisor en relación con el destinatario del mensaje (Spangardt; Ruth; Schramm, 2016).

A partir del marco teórico y de los estudios precedentes desarrollados, se plantean las siguientes preguntas de investigación: PI1: ¿Cuál es la presencia de los distintos tipos de testimoniales y cuál ha sido su evolución en la publicidad en radio? PI2: ¿Cuál es la presencia según tipos de testimoniales y tipo de mensaje, y cuál ha sido su evolución en la publicidad en radio?

PI3: ¿Cuál es la presencia según tipos de testimoniales y petición a la audiencia y cuál ha sido su evolución en la publicidad en radio?

\section{Metodología}

Se ha optado por una metodología de naturaleza cuantitativa y polietápica basada en la técnica del análisis de contenido que permite una descripción objetiva y sistemática del contenido manifiesto (Berelson, 1952) de cuñas publicitarias. En este caso se trata de dos muestras de testimoniales publicitarios protagonizados por portavoces cuya finalidad es trasladar al oyente los supuestos beneficios saludables de determinados productos, todo ello referido a la evolución de dicha presencia en los años 2009 y 2016.

\subsection{Unidades de análisis y muestra}

Siguiendo un criterio estratégico intencional, el mes elegido para la obtención de las muestras iniciales fue junio, por ser uno de los de mayor ocupación publicitaria según el estudio de inversión de referencia del sector Infoadex. Para la selección de las emisoras comerciales de mayor audiencia que emiten en español, de difusión nacional, se tomaron los datos de la oleada del EGM (Estudio general de medios) coincidentes con los primeros meses de los años analizados (AIMC, 2009; 2016). En ambos años las emisoras fueron las mismas excepto en dos casos.

Radio generalista:

- Cadena SER

- Onda Cero

- Cadena Cope

- Punto Radio sólo aparece en la muestra de 2009. 
Radio temática:

- C40

- Dial

- Europa FM

- C100

- Kiss FM

- Máxima FM

- Radio Olé

- M80

- Rock FM sólo aparece en la muestra de 2016.

\subsection{Procedimiento}

Se registraron las 24 horas de emisión de las 24 emisoras en cuatro semanas de lunes a viernes en días consecutivos (martes, miércoles y jueves) en un dispositivo de almacenamiento externo de memoria. Mediante un programa informático de audio (Sony Sound Forge Audio Studio 9.0) se analizaron las 576 horas (288 horas/año) de la programación emitida para detectar las cuñas publicitarias y configurar el corpus inicial del análisis. Se obtuvieron 1.664 en el año 2009 y 3.909 en 2016.

En primer lugar se desecharon las cuñas promocionales, es decir, las basadas en ofertas o cuyo beneficio es una recompensa económica y que por lo general son locutadas por una voz anónima no identificada que de no ser eliminadas hubieran sesgado los resultados.

En una segunda fase se seleccionaron los anuncios en los que se proponían supuestos beneficios saludables, es decir, que se vincularan al tratamiento, alivio o prevención de alguna enfermedad del tipo que sea, excluyendo los medicamentos que se dispensan sin receta. Por lo general, la publicidad de productos con supuesta finalidad sanitaria pertenece a cuatro categorías de producto (Perelló Oliver; Muela-Molina, 2011):

- alimentación;

- bebidas;

- belleza;

- higiene y salud.

Tras esta etapa, las muestras iniciales quedaron reducidas a 409 unidades en el año 2009 y 1.201 en 2016.

En la fase final se consideraron sólo los anuncios en los que el producto era descrito por un tercero que representa al anunciante, denominado portavoz. Tras este filtrado las muestras quedaron integradas por 147 anuncios en 2009 y 122 en 2016.

\subsection{Variables}

Las variables del estudio han sido operativizadas a partir del enunciado de la normativa analizada que regula la presencia de testimoniales en publicidad.

- Año. (1) 2009; (2) 2016.

- Tipo de portavoz. La voz protagonista del mensaje que encarna al anunciante se ha clasificado según los siguientes atributos (Belch; Belch, 2013; Fleck; Michel; Zeitoun, 2014; Friedman; Termini; Washington, 1976; Stafford; Stafford; Day, 2002): Famoso del mundo de la (1) Moda, (2) Cine, (3) Música, (4) Deporte, de las (5) Noticias y medios de comunicación; (6) Empleado de la empresa; (7) Experto profesional como doctor/a, enfermero/a o farmacéutico/a; (8) Consumidor.

- Tipo de testimonio. Esta variable está configurada a partir del rol que adopta el portavoz y su relación con el producto a la hora de describirlo (Kaphingst et al., 2004; Keel; Nataraajan, 2012; Seno; Lukas, 2007; Stern, 1991; Tulloch, 2014): (1) Presentación. De forma implícita, el producto, sus características y/o funciones se publicitan a través de un mensaje descriptivo con poca o ninguna implicación del portavoz con la marca, por lo que predomina el uso del sujeto en tercera persona; (2) Recomendación. De forma explícita, el testimonial recomienda el producto en base a sus características o beneficios y comparte con el oyente su experiencia positiva y conocimiento como consumidor, por lo que el grado de implicación es alto y se dirige a la audiencia en primera persona.

- Llamada a la acción. Como una forma de inducción al consumo, además de publicitar el producto, el portavoz le pide a la audiencia que lo adquiera, que lo compre, que llame por teléfono para informarse o que visite la web entre otros imperativos, por lo que esta variable consta de dos atributos (McCracken, 1989; Seno; Lukas, 2007): (1) Ausencia; (2) Presencia.

Para preservar la confiabilidad de las muestras, dos investigadores realizaron la codificación de las variables por separado. La única variable que generó algunas discrepancias fue la del "Tipo de testimonio" por la presencia de varios pronombres utilizados en un anuncio. En estos casos, el problema se resolvió codificando por la persona del sujeto que aparecía con más frecuencia. Asimismo, para la variable "Llamada a la acción" sólo se consideró el final del anuncio, mientras que para el tipo de portavoz y testimonio sólo se analizó el cuerpo de texto. 
Por último, se debe señalar que para el filtrado de las muestras, la codificación de las unidades muestrales, el análisis de frecuencias y los tests de significatividad relativos a cada cruce de variables, se utilizó el programa estadístico SPSS versión 17.

\section{Resultados}

Los resultados muestran el uso que muchos anunciantes realizan de testimonios de personas, en numerosos casos de reconocido prestigio, como líderes de opinión o supuestos profesionales de la sanidad cuando la legislación establece serias limitaciones y prohibiciones al respecto.

De la explotación de los resultados relativos a los dos años estudiados, y dando respuesta a la PI1, la tabla 1 constata que el perfil de portavoz más utilizado es el del 'Consumidor' $(55,78 \%$ en 2009 y $41,80 \%$ en 2016). Sin embargo, a lo largo de los últimos años se ha reforzado la presencia del 'Empleado de la empresa' anunciante como prescriptor de este tipo de productos, en detrimento principalmente de los portavoces vinculados a los informativos y los medios de comunicación, que han pasado de representar un $26,43 \%$ del total a un modesto $11,48 \%$. Dada la influencia que ejercen en la opinión pública, un dato destacable es la presencia de periodistas y personalidades de los medios, especialmente procedentes de la televisión, que en 2009 era el segundo portavoz más utilizado y en 2016 el tercero en preferencia.

En respuesta a la PI2 y desagregando por "Tipo de testimonio" (tabla 2), son también los portavoces de tipo “Consumidor' los que más uso hicieron de las presentaciones en 2009 , alcanzando un $47,5 \%$ del total como muestra la transcripción de la siguiente cuña:

Mujer 1: "Lucía, ¿tú sabes qué es Biosil del que todo el mundo habla?

Mujer 2: Biosil es un generador avanzado de colágeno.

Mujer 1: ¿Y eso qué es?

Mujer 2: Pues un producto que te ayuda a que tú generes tu propio colágeno y volver a verte como si tuvieras 20 años. Mujer 1: ¡Aaah eso me encanta! ¿Y funciona?

Mujer 2: ¿Que si funciona? En EUA ha sido premiado 7 años como el mejor producto.

Mujer 1: ¿Y dónde encuentro Biosil? En farmacias y parafarmacias".

Tabla 2. Evolución del tipo de portavoz y tipo de testimonio en la publicidad en radio 2009-2016.

\begin{tabular}{|c|c|c|c|c|c|c|c|c|c|c|c|c|}
\hline & \multicolumn{4}{|c|}{ Presentación } & \multicolumn{4}{|c|}{ Recomendación } & \multicolumn{4}{|c|}{ Valor estadístico } \\
\hline & \multicolumn{2}{|c|}{2009} & \multicolumn{2}{|c|}{2016} & \multicolumn{2}{|c|}{2009} & \multicolumn{2}{|c|}{2016} & \multicolumn{2}{|c|}{2009} & \multicolumn{2}{|c|}{2016} \\
\hline & $\mathbf{n}$ & $\%$ & $\mathbf{n}$ & $\%$ & $\mathbf{n}$ & $\%$ & $n$ & $\%$ & $x^{2}$ & $\mathbf{p}$ & $x^{2}$ & $\mathbf{p}$ \\
\hline \multicolumn{13}{|l|}{ Cine } \\
\hline Música & & & 6 & 9,38 & & & & & & & 5,719 & 0,017 \\
\hline \multicolumn{13}{|l|}{ Deporte } \\
\hline \multicolumn{13}{|l|}{ Moda } \\
\hline $\begin{array}{l}\text { Noticias y medios de } \\
\text { comunicación }\end{array}$ & 4 & 10,00 & 2 & 3,13 & 35 & 32,71 & 12 & 20,69 & 7,704 & 0,006 & 9,240 & $0,002^{\mathrm{a}}$ \\
\hline Empleado de la empresa & 1 & 2,50 & 45 & 70,31 & & & & & 2,693 & 0,101 & 64,614 & $0,001^{a}$ \\
\hline Experto profesional & 16 & 40,00 & 5 & 7,81 & 9 & 8,41 & 1 & 1,72 & 22,584 & $0,001^{\mathrm{a}}$ & 2,412 & 0,120 \\
\hline Consumidor & 19 & 47,50 & 6 & 9,38 & 63 & 58,88 & 45 & 77,59 & 1,528 & 0,216 & 58,190 & $0,001^{a}$ \\
\hline $\begin{array}{l}\text { Total (N 2009=147; N } \\
2016=122)\end{array}$ & 40 & 100 & 64 & 100 & 107 & 100 & 58 & 100 & & & & \\
\hline
\end{tabular}

a Cruce estadísticamente significativo 
En 2016 este porcentaje descendió al 9,38\%, siendo el 'Empleado de la empresa' el tipo de portavoz que adquirió más protagonismo entre esa modalidad como muestra el siguiente ejemplo:

"Presentamos Puleva digestiva, la que mejor te sienta por su bajo contenido en lactosa. Para todas aquellas personas que quieren disfrutar de la leche. Puleva digestiva, seguro que te sienta bien".
La presencia de determinados testimoniales en publicidad se ha convertido en un objeto muy regulado por parte de la administración pública dado su potencial persuasivo

Las recomendaciones han utilizado mayoritariamente la figura del 'Consumidor' -58,88\% en 2016 y 77,59\% en 2016 para trasladar sus mensajes como ilustra la siguiente transcripción:

"Yo tomo Be Vegan, el batido de proteínas vegetales de fácil digestión que me aporta fibra, probióticos, enzimas y super alimentos. Be Vegan ayuda a incrementar mi masa muscular y es el complemento perfecto de mi dieta de adelgazamiento".

Otro dato destacable es que los periodistas y profesionales de los medios son los segundos portavoces que más avalan el producto en primera persona compartiendo así su experiencia con el oyente como puede leerse en el ejemplo de la siguiente cuña:

“¿Sabías que el cansancio y el estrés puede disminuir tu deseo sexual?

Soy Manolo Lama y te recomiendo Energisil vigor. Energisil te devuelve la libido y el deseo sexual. Recupera el deseo con Energisil vigor".

Aunque la presencia se ha reducido en 2016 no deja de ser un dato que reclama poderosamente la atención.

La tabla 3 muestra la presencia de peticiones expresas a la audiencia de actuar de una determinada manera a favor del anunciante, desagregadas por los tipos de portavoces analizados. Respondiendo a la PI3, se aprecia cómo en 2009 esas peticiones imperativas recaen mayoritariamente sobre el portavoz que ejerce el rol de consumidor $(66,07 \%)$, mientras que en 2016 son los empleados de la empresa que, a la vez que han adquirido un mayor protagonismo relativo en la muestra, asumen esa función imperativa de manera mayoritaria $(47,13 \%)$ como puede leerse en la transcripción de la siguiente cuña:

(...) "te lo entregamos en tu domicilio llamando al 902303033 (...). Date prisa, llama al 902303033 y obtendrás unos placenteros resultados. The sensual tea es un producto increíble. 9023030 33".

Con todo, los portavoces que ejercen de supuestos consumidores siguen teniendo un peso muy significativo en la incitación al consumo (42,53\%), como ilustra el siguiente ejemplo:

"Mi chico y yo tomamos Adipesina. Es de base vegetal de Phytovit. Pídelo en farmacias y centros de dietética y adiós a los michelines".

Tabla 3. Evolución del tipo de portavoz y llamada a la acción en la publicidad en radio 2009-2016.

\begin{tabular}{|c|c|c|c|c|c|c|c|c|}
\hline & \multicolumn{4}{|c|}{ Petición audiencia } & \multicolumn{4}{|c|}{ Valor estadístico } \\
\hline & \multicolumn{2}{|c|}{2009} & \multicolumn{2}{|c|}{2016} & \multicolumn{2}{|c|}{2009} & \multicolumn{2}{|c|}{2016} \\
\hline & $\mathbf{n}$ & $\%$ & $\mathbf{n}$ & $\%$ & $x^{2}$ & $\mathbf{p}$ & $x^{2}$ & $\mathbf{p}$ \\
\hline \multicolumn{9}{|l|}{ Cine } \\
\hline Música & & & 6 & 6,90 & & & 2,539 & 0,111 \\
\hline \multicolumn{9}{|l|}{ Deporte } \\
\hline \multicolumn{9}{|l|}{ Moda } \\
\hline Noticias y Medios de Comunicación & 7 & 12,50 & 2 & 2,30 & 9,136 & $0,003^{\mathrm{a}}$ & 25,138 & $0,001^{\mathrm{a}}$ \\
\hline Empleado de la empresa & 1 & 1,79 & 41 & 47,13 & 1,636 & 0,201 & 13,662 & $0,001^{\mathrm{a}}$ \\
\hline Experto profesional & 11 & 19,64 & 1 & 1,15 & 0,445 & 0,505 & 9,211 & $0,002^{\mathrm{a}}$ \\
\hline Consumidor & 37 & 66,07 & 37 & 42,53 & 3,883 & 0,049 & 0,066 & 0,798 \\
\hline Total (N 2009=147; N 2016=122) & 56 & 100 & 87 & 100 & & & & \\
\hline
\end{tabular}

a Cruce estadísticamente significativo

\section{Discusión y conclusiones}

Este estudio analiza la presencia de testimonios en publicidad cuando supuestamente la legislación vigente no lo permite. Aunque estudios previos han mostrado dicha presencia ilícita (Perelló-Oliver; Muela-Molina, 2011), esta investigación profundiza en la modalidad de las personas que en nombre del anunciante presentan o recomiendan un producto. Los hallazgos proporcionan una novedosa visión del uso que los anunciantes hacen de la personificación y la naturaleza 
de dicha utilización en un medio que no suele ser de preferencia para los investigadores a pesar del enorme impacto que tiene en términos de audiencia y credibilidad.

Una de las conclusiones a destacar es la escasa presencia de famosos/as en favor de la figura del consumidor. Ello se debe a que la radio, al carecer de imagen, no es un medio que permita rentabilizar la inversión requerida para aprovechar el atractivo de una celebridad. Y, por otro lado, cabe destacar el predominio de la figura del consumidor que, situándose al mismo nivel que el oyente, se dirige a él como si de un amigo se tratara con quien comparte problemas cotidianos que le ayuda a resolver. Sin embargo, la mayoría de estos portavoces no son ni si quiera testimonios reales.

Desde la perspectiva de la administración pública, se extraen varias implicaciones del presente estudio. Los controles legales sobre la publicidad de productos relacionados con la salud deben ser mucho más efectivos ya que, como también han mostrado estudios previos (Perelló Oliver; Muela-Molina; Hormigos-Ruiz, 2016), marcas cuyos anuncios son objeto de reclamación año

\section{El sistema de autorregulación publicita- ria en España es deficiente en el control de la publicidad ilícita de productos que proponen supuestos beneficios para la salud} tras año ante los organismos autorreguladores siguen siendo emitidos con total impunidad. Al fraude económico hay que sumar el riesgo en la salud que pueden provocar productos que no tienen una base científica que garantice y verifique sus cualidades. Además de un mayor seguimiento y vigilancia de la publicidad de los productos con supuesta finalidad sanitaria, también sería pertinente realizar controles más severos respecto de su fabricación y distribución ya que muchas empresas operan bajo la fórmula fly-by-night (Peiró-Abásolo, 2006). Se trata de sociedades que tienen como principal público objetivo a personas especialmente sensibles o vulnerables a este tipo de engaños animándoles con repeticiones constantes de sus supuestas bondades cuyo ejemplo extremo son los llamados "productos milagro".

También esencial es la realización de campañas educativas tanto por parte de los organismos públicos como los de autorregulación tal y como se insta desde la Comisión Europea. Ello incrementaría el conocimiento por parte de la población en general y del consumidor para evaluar campañas irresponsables que pueden afectar su comportamiento de consumo, e incluso su salud. En consecuencia, las reclamaciones presentadas en este ámbito ante la asociación de autorregulación también aumentarían en base a la mayor concienciación de la población para defender sus derechos como consumidores (González-Esteban; Feenstra, 2018). Por tanto, la educación mediática y la autorregulación de los medios, en especial los audiovisuales, requieren políticas de implementación no sólo en la televisión, que es el medio más vigilado, sino también en otros como la radio, que cuenta con la segunda mayor audiencia y con la mayor credibilidad informativa.

Aunque el anunciante es el máximo responsable de que algunos testimonios sean engañosos o sean susceptibles de inducir a engaño, la responsabilidad de que esos mensajes lleguen a su destinatario también debe extenderse a las agencias de publicidad que los hacen y al medio que los difunde (Presas-Mata, 2018; Vilajoana-Alejandre; Rom-Rodríguez, 2017). En este caso, dada la credibilidad que la audiencia otorga a la radio, el hecho de que un anuncio sea aceptado para ser emitido puede ser tomado por el oyente como un aval o garantía, ya que se trata de un medio al que le confiere una gran confianza para mantenerse informado. Para ello sería deseable que tanto editores como medios desarrollaran una política de publicidad y pre-autorización (pre-clearance) comercial para controlar voluntariamente los anuncios que puedan parecer ilícitos (Kertz; Ohanian, 1992) antes de su emisión evitando, así, el posible perjuicio que pudiera producir en la audiencia.

Una persona de confianza es alguien que se percibe como honesto y sincero. Por tanto, la responsabilidad con el consumidor también ha de ser extensible al portavoz que habla en representación del anunciante (FTC, 2009). Tanto las personas famosas, celebridades o personajes de gran reconocimiento y prestigio social como los expertos en salud y profesionales sanitarios o farmacéuticos que respaldan un producto deben autoexigirse pleno conocimiento y validez del testimonio que van a dar, para lo que deberán haber testado el producto para acumular una experiencia fiable (Kertz; Ohanian, 1992). A mayor abundamiento, como uno de los factores más influyentes en el proceso de persuasión (Petty; Cacioppo; Schumann, 1983), un portavoz en quien el oyente confíe puede provocarle actitudes más irreflexivas respecto del contenido del mensaje (Priester; Petty, 2003).

Ello es de especial importancia en los casos en los que el portavoz es un líder de opinión e influye en la formación y mantenimiento de la opinión pública ostentando una credibilidad y confianza que se proyecta, no sólo a las noticias o temas que trata en los medios de comunicación, sino también a los productos que avala. Casos destacados que tuvieron una gran repercusión social como la conocida marca de yogur Actimel de Danone recomendada por una prestigiosa periodista de televisión reclamada como publicidad engañosa por declarar falsos beneficios saludables (Muela-Molina, 2014), u otros prescritos por destacados presentadores de la televisión, actores o deportistas, siguen anunciándose reiteradamente con total impunidad, vulnerando la normativa que prohíbe la presencia de estos testimoniales. 


\section{Referencias}

AIMC (2009). Resumen general del EGM (Estudio general de medios) abril 2008 a marzo 2009. Asociación para la Investigación de Medios de Comunicación.

https://www.aimc.es/a1mc-c0nt3nt/uploads/2010/09/resumegm109.pdf

AIMC (2016). Resumen general del EGM (Estudio general de medios) abril 2015 a marzo 2016. Asociación para la Investigación de Medios de Comunicación.

https://www.aimc.es/a1mc-c0nt3nt/uploads/2016/04/resumegm116.pdf

Amos, Clinton; Holmes, Gary; Strutton, David (2008). "Exploring the relationship between celebrity endorser effects and advertising effectiveness". International journal of advertising, v. 27, n. 2, pp. 209-234.

https://doi.org/10.1080/02650487.2008.11073052

Belch, Georges E.; Belch, Michael A. (2013). "A content analysis study of the use of celebrity endorsers in magazine advertising". International journal of advertising, v. 32, n. 3, pp. 369-389.

https://doi.org/10.2501/IJA-32-3-369-389

Berelson, Bernard (1952). Content analysis in communication research. New York: Hafner Press.

Biswas, Dypayan; Biswas, Abhijit; Das, Neel (2006). "The differential effects of celebrity and expert endorsements on consumer risk perceptions. The role of consumer knowledge, perceived congruency, and product technology orientation". Journal of advertising, v. 35, n. 2, pp. 17-31.

https://doi.org/10.1080/00913367.2006.10639231

Buchholz, Laura M.; Smith, Robert E. (1991). "The role of consumer involvement in determining cognitive response to broadcast advertising". Journal of advertising, v. 20, n. 1, pp. 4-17.

https://doi.org/10.1080/00913367.1991.10673202

Doss, Samuel (2011). "The transference of brand attitude: the effect on the celebrity endorser". Journal of management and marketing research, v. 7, n. 1, pp. 58-70.

http://www.aabri.com/manuscripts/10636.pdf

Eisend, Martin; Langner, Tobias (2010). “Immediate and delayed advertising effects of celebrity endorsers' attractiveness and expertise". International journal of advertising, v. 29, n. 4, pp. 527-546.

https://doi.org/10.2501/S0265048710201336

España (1996). "Real decreto 1907/1996, de 2 de agosto, sobre publicidad y promoción comercial de productos, actividades o servicios con pretendida finalidad sanitaria". BOE, n. 189, 6 agosto, pp. 24322-24325.

https://www.boe.es/buscar/doc.php?id=BOE-A-1996-18085

España (2011). “Ley 17/2011, de 5 de julio, de seguridad alimentaria y nutrición”. BOE, n. 160, 6 julio.

https://www.boe.es/buscar/act.php?id=BOE-A-2011-11604

FTC (2009). Guides concerning the use of endorsements and testimonials in advertising. Federal Acquisition Regulation (16 CFR Part 255); Final Rule. Federal Trade Commission.

https://www.ftc.gov/sites/default/files/documents/federal_register_notices/guides-concerning-use-endorsementsand-testimonials-advertising-16-cfr-part-255/091015guidesconcerningtestimonials.pdf

Fleck, Nathalie; Michel, Géraldine; Zeitoun, Valérie (2014). "Brand personification through the use of spokespeople: An exploratory study of ordinary employees, CEOs, and celebrities featured in advertising". Psychology \& marketing, v. 31, n. 1, pp. 84-92.

https://doi.org/10.1002/mar.20677

Friedman, Hershey H.; Termini, Salvatore; Washington, Robert (1976). "The effectiveness of advertisements utilizing four types of endorsers". Journal of advertising, v. 5, n. 3, pp. 22-24.

https://doi.org/10.1080/00913367.1976.10672647

Gaied, Aïcha-Meksi; Ben-Rached, Kaouther-Saied (2010). "The persuasive effectiveness of famous and non-famous endorsers in advertising". Ibima business review, ID: 474771.

https://doi.org/10.5171/2010.474771

González-Esteban, Elsa; Feenstra, Ramón (2018). “Ética y autorregulación publicitaria: perspectiva desde las asociaciones de consumidores". El profesional de la información, v. 27, n. 1, pp. 106-114.

https://doi.org/10.3145/epi.2018.ene.10

Hastak, Manoj; Mazis, Michael B. (2011). “Deception by implication: A typology of truthful but misleading advertising and labeling claims". Journal of public policy \& marketing, v. 30, n. 2, pp. 157-167.

https://doi.org/10.1509/jppm.30.2.157 
Kaphingst, Kimberly A.; Dejong, William; Rudd, Rima E.; Daltroy, Lawren H. (2004). "A content analysis of direct-to-consumer television prescription drug advertisements". Journal of health communication, v. 9, n. 6, pp. 515-528. https://doi.org/10.1080/10810730490882586

Keel, Astrid; Nataraajan, Rajan (2012). "Celebrity endorsements and beyond: New avenues for celebrity branding". Psychology \& marketing, v. 29, n. 9, pp. 690-703.

https://doi.org/10.1002/mar.20555

Kertz, Consuelo-Lauda; Ohanian, Roobina (1992). "Source credibility, legal liability, and the law of endorsements". Journal of public policy \& marketing, v. 11, n. 1, pp. 12-23.

https://doi.org/10.1177/074391569201100102

Khatri, Puja (2006). “Celebrity endorsement: A strategic promotion perspective”. Indian media studies journal, v. 1, n. 1, pp. 25-37. https://satishserial.com/issn0972-9348/finaljournal03.pdf

La-Tour, Caroline; Smith, Mickey (1986). "A study of expert endorsement of OTC pharmaceutical products". Journal of pharmaceutical marketing \& management, v. 1, n. 2, pp. 117-128.

https://doi.org/10.3109/J058v01n02_17

Limbu, Yam B.; Huhmann, Bruce A.; Peterson, Robin T. (2012). "An examination of humor and endorser effects on consumers' responses to direct-to-consumer advertising: The moderating role of product involvement". International journal of pharmaceutical and healthcare marketing, v. 6, n. 1, pp. 23-38.

https://doi.org/10.1108/17506121211216888

McCracken, Grant (1989). "Who is the celebrity endorser? Cultural foundations of the endorsement process". Journal of consumer research, v. 16, n. 3, pp. 310-321.

https://doi.org/10.1086/209217

Muela-Molina, Clara (2014). “Misleading advertising in food products”. En: Sánchez-Hernández, María-Filomena; Jiménez-Morales, Mónika; Carrillo-Durán, María-Victoria. Communication and body image. México: Pearson. ISBN: 978607 3212434

https://www.researchgate.net/publication/273832390_Misleading_Advertising_in_Food_Products

Muela-Molina, Clara; Martín-Santana, Josefa-Delia; Reinares-Lara, Eva (2018). “Journalists as radio advertising endorsers in news or talk radio stations". Journalism.

https://doi.org/10.1177/1464884917753785

Ohanian, Roobina (1990). "Construction and validation of a scale to measure celebrity endorsers' perceived expertise, trustworthiness, and attractiveness". Journal of advertising, v. 19, n. 3, pp. 39-52.

https://doi.org/10.1080/00913367.1990.10673191

Peiró-Abásolo, Andrea (2006). “La publicidad de los Ilamados 'productos milagro””. Estudios sobre consumo, v. 78, pp. 21-31.

Perelló-Oliver, Salvador; Muela-Molina, Clara (2011). "An analysis of health-related advertising in Spanish radio". Cuadernos de información, v. 29, pp. 67-76.

http://www.cuadernos.info/index.php/CDI/article/download/237/230

Perelló-Oliver, Salvador; Muela-Molina, Clara (2016). El sistema de autorregulación publicitaria en España desde la perspectiva de la protección del consumidor. Prisma social, n. 17, pp. 24-45.

http://revistaprismasocial.es/article/view/1274/1337

Perelló-Oliver, Salvador; Muela-Molina, Clara; Hormigos-Ruiz, Jaime (2016). “Publicidad ilícita, productos saludables y autorregulación". Cuadernos.info, n. 38, pp. 51-67.

https://doi.org/10.7764/cdi.38.922

Petty, Richard E.; Cacioppo, John T.; Schumann, David (1983). “Central and peripheral routes to advertising effectiveness: The moderating role of involvement". Journal of consumer research, v. 10, n. 2, pp. 135-146.

https://doi.org/10.1086/208954

Presas-Mata, Fátima (2018). "La responsabilidad social de los stakeholders en la publicidad: necesidad de un compromiso ético en la industria publicitaria". Methaodos. Revista de ciencias sociales, v. 6, n. 1, pp. 38-51.

https://doi.org/10.17502/m.rcs.v6i1.208

Priester, Joseph R.; Petty, Richard E. (2003). "The influence of spokesperson trustworthiness on message elaboration, attitude strength, and advertising effectiveness". Journal of consumer psychology, v. 13, n. 4, pp. 408-421.

https://doi.org/10.1207/S15327663JCP1304_08 
Seno, Diana; Lukas, Bryan A. (2007). "The equity effect of product endorsement by celebrities: A conceptual framework from a co-branding perspective". European journal of marketing, v. 41, n. 1/2, pp. 121-134.

https://doi.org/10.1108/03090560710718148

Silvera, David H.; Austad, Benedikte (2004). "Factors predicting the effectiveness of celebrity endorsement advertisements". European journal of marketing, v. 38, n. 11/12, pp. 1509-1526.

https://doi.org/10.1108/03090560410560218

Spangardt, Benedikt; Ruth, Nicholas; Schramm, Holger (2016). “'...And please visit our Facebook page, too!' How radio presenter personalities influence listeners' interactions with radio stations". Journal of radio \& audio media, v. 23, n. 1, pp. 68-94.

https://doi.org/10.1080/19376529.2016.1155710

Stafford, Marla-Royne; Stafford, Thomas F.; Day, Ellen (2002). "A contingency approach: The effects of spokesperson type and service type on service advertising perceptions". Journal of advertising, v. 31, n. 2, pp. 17-34.

https://doi.org/10.1080/00913367.2002.10673664

Stern, B. Bárbara (1991). "Who talks advertising? Literary theory and narrative 'point of view'”. Journal of advertising, v. 20 , n. 3, pp. 9-22.

https://doi.org/10.1080/00913367.1991.10673344

Tulloch, John (2014). "Ethics, trust and the first person in the narration of long-form journalism". Journalism, v. 15, n. 5, pp. $629-638$.

https://doi.org/10.1177/1464884914523233

Vilajoana-Alejandre, Sandra; Rom-Rodríguez, Josep (2017). "Sistema de autorregulación publicitaria: del compromiso ético al control efectivo de la publicidad en España”. El profesional de la información, v. 26, n. 2, pp. $192-200$.

https://doi.org/10.3145/epi.2017.mar.05

Wu, Wann-Yih; Linn, Cho-Thwe; Fu, Chen-Su; Sukoco, Badri-Munir (2012). "The role of endorsers, framing, and rewards on the effectiveness of dietary supplement advertisements". Journal of health communication, v. 17, n. 1, pp. 54-75.

https://doi.org/10.1080/10810730.2011.585689

\section{Colección EPI Scholar}

\section{Libros científicos de Información, Documentación y Comunicación}

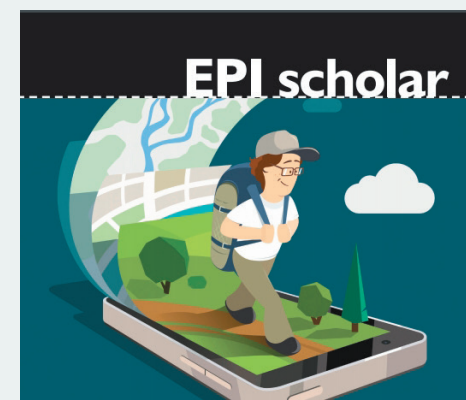

TURISMO RURAL ONLINE PÁGINAS WEB Y REDES SOCIALES

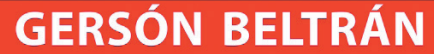

información- SII EDITORIAL UOC

\section{Turismo rural online. Páginas web y redes sociales de Gersón Beltrán}

Los turistas están conectados en todo momento y se mueven en un entorno social, local y móvil: buscan información en internet antes de ir a su destino y realizan las reservas por anticipado, utilizan el móvil durante su experiencia turística para comunicarse y, tanto durante como tras el viaje, comparten sus opiniones en medios sociales.

Las empresas y destinos turísticos deben adaptarse a esta realidad, en la que lo físico es inseparable de lo online, conformando un entorno híbrido. Paralelamente, los espacios rurales sufren numerosos problemas de desarrollo y despoblación, y el turismo rural constituye una actividad que puede ayudar a revertir esta situación. Las nuevas tecnologías se configuran como herramientas que unen a los turistas conectados con estos espacios.

Este libro ofrece una visión general de la situación del turismo rural online, así como las claves para que las empresas y destinos turísticos logren conectar con los turistas y éstos disfruten de las experiencias únicas que les ofrece el turismo rural.

Beltrán, Gersón (2019). Turismo rural online. Páginas web y redes sociales. Barcelona: El profesional de la información, Editorial UOC, colección EPI Scholar n. 11, 170 pp. ISBN: 9788491803881

\section{Información}

http://www.elprofesionaldelainformacion.com/librosEPIScholar.html 